\title{
Strategies to Enhance Antimicrobial Resistance Stewardship
}

\section{Alanazi MQ*}

Drug Policy and Economic Center, King Abdulaziz Medical City, Ministry of National Guard-Health Affairs, Riyadh, Saudi Arabia

*Corresponding author: Prof. Menyfah Q. Alanazi, Drug Policy \& Economic Centre, King Abdulaziz Medical City, Ministry of National Guard-Health Affairs, Riyadh, Saudi Arabia, Tel: +966118011111; E-mail: Dr.monifah@gmail.com

Received date: December 8, 2017; Accepted date: January 12, 2018; Published date: January 15, 2018

Copyright: $\odot 2018$ Alanazi MQ et al. This is an open-access article distributed under the terms of the Creative Commons Attribution License, which permits unrestricted use, distribution, and reproduction in any medium, provided the original author and source are credited.

\begin{abstract}
Antibiotics are widely prescribed therapeutic agents given as management for a range of bacterial infections. The development of antibiotic resistance is one of the most challenging problems in world. It occurs due to over use or inappropriate use of antibiotic. It occurs when the bacteria change in a way that reduces or stops the effectiveness of antibiotics, then the bacteria can survive and continue to grow and strengthen. Antibiotic resistance is accelerated by the misuse and overuse of antibiotics, as well as poor infection prevention and control. Steps to reduce the impact and limit the spread of resistance such as education of health care professionals, patient counselling and maker policies.
\end{abstract}

Keywords: Antibiotics; Antimicrobial drugs; Multidrug resistance

\section{Introduction}

In managing and preventing bacterial infections, Antibiotics or Antimicrobial drugs are prescribed by healthcare providers to either exterminate (bactericidal) or inhibit growth of susceptible bacteria, rather than killing them immediately; will eventually lead to bacterial death (bacteriostatic) [1]. It works against infections caused by bacteria which are less common than viral infection. Thus, it is not advised that it be prescribed to treat diseases caused by viral infection, such as common cold, influenza or flu, coughs and bronchitis, sore throats and acute sinusitis. If antibiotics or antimicrobials are overused and inappropriately, the result may lead to antibiotic resistance.

Antibiotics or Antimicrobial drugs were discovered in the 1940's to treat patients with infectious diseases. It greatly reduced said diseases and death rate was lowered, if not totally evaded. Patients who were hospitalized because of severe bacterial infections were initially injected with a broad-spectrum Antibiotic intravenously for 48 hours. A laboratory test will be done to confirm the infecting bacteria and appropriate Antibiotic drug will be prescribed for treatment of the particular disease [2]. If ever the patient would clinically improve, there would be changes in the drug dosage and drug form, from IV to oral. Nonetheless, it came to a point when the Antibiotic drugs became less effective due to long period of time the patient used it. The infectious organisms intended to be eradicated by the use of Antibiotic drugs have adapted to patients, making the latter less effective [3].

\section{Categorization of antibiotics (classifications)}

Antibiotics or Antimicrobials drugs are classified in accordance to their mechanism of action, chemical structure and spectrum of activity $[1,4]$.

\section{Categorization is based on chemical structure}

In chemical structure, Antibiotics drugs generally show the same configuration of antibacterial activity, effectiveness, toxicity and allergic potential. The main classifications are as follows:
a. Beta-Lactams (Penicillin \& ampicillin, Cephalosporin)
b. Macrolides
c. Fluroquinolones
d. Tetracycline and
e. Aminoglycoside

Each class consists of a range of drugs but each one is still distinctive in its own way.

\section{Categorization is based on mechanism of action}

a. Inhibit cell wall formation: Penicillins and Cephalosporins.

b. Inhibit cell Membrane Function: Polymyxins, the cell membrane

c. Interfere with DNA formation: Rifamycins, Lipiarmycins, Quinolones and Sulfonamides which have bactericidal activities

d. Block protein formation: Macrolides, Aminoglycosides, Lincosamides and Tetracyclines.

\section{Categorization is based on spectrum of activity}

There are two types of Antibiotics, to wit:

a. Narrow-spectrum Antibiotic: It targets a specific type of bacteria, i.e. gram- negative or gram-positive, which is effective against specific families of bacteria.

b. Broad-spectrum Antibiotic: It acts against a wide range of disease-causing bacteria.

Four (4) new classes of antibacterial compounds have been discovered in the late 2000s and early 2010s which are cyclic lipopeptides (such as daptomycin), glycylcyclines (such as tigecycline), oxazolidinones (such as linezolid), and lipiarmycins (such as fidaxomicin) [5]. 


\section{Scope of the Problem}

Resistance to antibiotics represents an alarming and rising predicament because some infectious diseases are becoming tougher to treat. Resistant bacteria do not respond to antibiotics and continue to aggravate illness, at times, cause death for the patient. It is one of the most challenging problems in world due to overuse or inappropriate use of antibiotic.

The main objective of the study is to promote awareness and understanding of antimicrobial resistance in order to overcome or eliminate antimicrobial resistance, minimize the cost of antibiotics and to optimize the use of antimicrobial drugs.

\section{Misuses of Antibiotics}

Eighty percent (80\%) of Antimicrobials are prescribed in Primary Care, due to common infections, i.e., sore throat, common cold, acute otitis media, acute infective conjunctivitis, acute bronchitis and acute sinusitis [6]. There are indications that antibiotics prescribed in said conditions has restricted results and can instead lead to adverse effects, increased or additional consultation costs, high costs of drugs and an increased risk of resistance $[7,8]$.

The inappropriate use of antibiotics is clearly shown in the following situations [3]:

a. Extended antimicrobial treatment without clear evidence of infection

b. Management of a positive clinical culture in the nonexistence of disease

c. Failure to constrict antimicrobial therapy when a relevant organism is recognized

\section{d. Continued prophylactic therapy}

e. Excessive use of certain antimicrobial agents

\section{Appropriateness of Prescribing Antibiotic}

Incorrect antibiotic prescribing is common among primary health care providers. Incorrect means wrong selection of antibiotics in the treatment of a particular illness, wrong dosage of drug, wrong frequency and wrong duration of time the drug will be consumed. Various researches conducted in and outside Europe had shown that over $40 \%$ of antibiotic prescriptions were more or less unsuitable for a particular illness. In a study conducted in Saudi Arabia, the prevalence of inappropriate ATB prescriptions with at least one or more types of errors was $46.2 \%$, considerably higher among pediatrics $(57.8 \%)$ compared to adults (38.7\%). Commonly occurrence of errors was the in the selection of proper ATB class (2.0\%), followed by frequency (4.2\%), dosage (21.7\%), and duration (28.6\%). Pediatrics were significantly of higher incidence in dosage and duration errors, whereas adults showed significantly higher in the selection errors $[9,10]$.

\section{Antibiotic Resistance}

Antimicrobial resistance is a major public health threat of the $21^{\text {st }}$ Century. Its bacteria could be extended to family members and peers and could even be a threat to the entire community. Antibioticresistant bacteria are often more difficult to eliminate and more expensive to manage, or even in some instance, it can lead to serious.
In some cases, the antibiotic-resistant infections can lead to serious infirmity or even death.

Antibiotic resistance is the capability of the bacteria to resist the properties or effects of an antibiotic to a particular disease. It occurs when the bacteria reduce or stops the efficacy of antibiotics, and survives (continues to grow and fortifies). Bacteria can become unaffected to antibiotics through numerous ways: (a) Some bacteria can "neutralize" an antibiotic by changing it in a mode that makes it harmless, (b) others have learned how to thrust the drug back outside of the bacteria before it can do any harm and (c) some type of bacteria can change their outer structure so the antibiotic has no method to attach to the bacteria it is designed to kill [2].

When bacteria are exposed to the same medication for a longer period of time, the bacteria are no longer affected by the drug. Patients who take antibiotic not suitable for their illness or do not need it for their illness, may not experience the efficacy of the drug. On the other hand, if they do not observe to take the full course of the prescribed drug, there is a greater chance for the bacteria to become resistant in future management of the illness because of exposure of the remaining bacteria to the antibiotic and may result a build-up resistance to the drug. It is reiterated that patients should complete the course of treatment for best outcome.

Bacteria are becoming resistant quicker than the development of advanced antibiotics which intimidates the ability of medical researchers to discover appropriate and highly valuable cures for management of particular bacteria and finite resources.

In reference to the Centers for Disease Control \& Prevention (CDC), approximately 2 million people in the United States acquired bacterial infections during admission to the hospital annually, and over $70 \%$ of the bacteria that cause these infections are resistant to at least one of the drugs normally used for treatment. These drug-resistant bacteria, such as methicillin-resistant Staphylococcus aureus (MRSA) and Pseudomonas aeruginosa, also strains of Klebsiella pneumonia, have developed resistance to all available beta-lactams $[2,8,11,12]$ World Health Organization (WHO) estimates a $3.7 \%$ of new cases world-wide and $20 \%$ of previously treated cases for MDR-TB $[2,6,8]$.

\section{Cost}

The cost of antibiotic increases annually because the demand of its consumption increases; consumption of antibiotics increases due to increase of antibiotic resistance (over use or inappropriate use of antibiotics). As antibiotic resistance increases in cost, mortality also increases [13].

The "cost" of an antibiotic drug is dependent on many factors such as the purchase price, administrative cost, prolonged hospitalization as a consequence of adverse effects, the cost of serum concentration monitoring, cost for culture, microbial sensitivity, and gram Positive, visits and consultation with physicians, and clinical efficacy [3].

When infections can no longer be treated by first-line antibiotics, more expensive drugs are prescribed. The longer duration treating an illness, longer hospital confinement, increases healthcare costs as well as the economic burden on families and societies. Antibiotic resistance (AMR) including multidrug resistance (MDR), are on the rise among many microorganisms in healthcare facilities as well as in community [13]. Reported data suggests that almost 2 million cases of infections are resistant. 
Bacterial resistance leading to $\$ 20$ Billion incremental direct healthcare cost [14] Estimates of European Medicines Agency (EMA) and European Centre for Disease Prevention and Control (ECDC) reported a toll of 25,000 deaths per year as a direct consequence of a MDR Infection with total cost of $€ 1.5$ billion [15]. In Canada, hospitalization caused by Resistant infections resulted in higher economic burden with excess cost of $\$ 9-\$ 14$ million $[8,13]$.

\section{Role of the Pharmacist}

Pharmacists perform an increasingly important role in enhanced antibiotic control, a vital component of antibiotic stewardship [16]. As a cornerstone in patient care and involved in all hospital teams that are responsible for development of antibiotic utilization in the hospital from the beginning, planning \& purchasing, added to hospital formulary, creation of Committees in prepare the guidelines, consultation, monitoring, until in the dispensing of medication to patients. Pharmacist participates in other jobs such as educating healthcare providers and patients. They play a critical role in coordinating better strategies to complement antibiotic stewardship, where they are directly working with medical and nursing staff to safeguard that patients are prescribed the best management by recommending on drug selection, dose, and administration method. They participate in local and national committee to monitor impact of antibiotic resistance and continuous education on relevant medical updates to healthcare providers and patients about the appropriate use of antibiotics and provide counselling to patients $[2,17]$.

\section{Recommendation for minimizing antibiotic resistance}

Educating people and periodically giving those current updates on antibiotic resistance will prevent and control said problem.

\section{Recommendation for individuals}

Patient education is the process by which pharmacists and healthcare providers deliver information to patients and their caregivers to improve their health $[7,8,16]$ :

1. Use antibiotics when prescribed by a physician.

2. Every time follow the instructions of healthcare professional who prescribed antibiotics.

3. Wash hands properly and frequently to avoid infections.

4. Make food hygienically, following the WHO Five Keys to Safer Food (possess clean,

5. Separate raw and cooked, cook thoroughly, store food at safe temperatures, use clean water and raw materials) and choose foods that have been produced without the use of antibiotics for growth promotion or disease prevention in healthy animals.

6. Avoiding close contact with infected people.

7. Preserve a healthy lifestyle by eating a balanced diet, drinking enough water, exercising, and getting enough rest.

8. Never take antibiotics prescribed for other patient, even with the same symptoms.

9. Do not take antibiotics that were extra from a previous infection.

10. Complete antibiotics course even if you feel better.
11. Discuss with pharmacist whether drug-drug interaction and food-drug interaction.

12. Store antibiotics properly. Stored at room temperature in a dry place, some require refrigeration such as reconstitution (solution).

\section{Recommendation for the pharmacist}

Provide clear medication information, orally and in written form to patients and/or their family on prescription instructions, advice on side effects, precautions, storage, diet and life style modifications.

1. Inform the patient specific directions on the use of antibiotic: route, frequency, duration, missed doses, and storage of antibiotics.

2. Use the "FRAIS" mnemonic when counselling the patients:

a. F Finish the course: Advise the patient to complete the course of antibiotic.

b. R Regular intervals (e.g. Six-hourly, eight-hourly, etc.)

c. A inform the patient when taken the antibiotic "After, with or before meal".

d. I Interactions, caution the patients about drug-drug interaction, drug-food interaction.

e. S Side effects, inform the patients side effects of drug such as diarrhoea.

3. Inform the patient the proper use of antibiotic.

\section{Recommendation for health care professionals}

Healthcare professionals can stop the spread of antibiotic resistance by:

1. Prescribing an antibiotic only when the patient needs it.

2. Prescribing the right antibiotic that targets the bacteria that is most likely causing their patient's illness.

3. Encouraging patients to consume the full dosage of the antibiotic prescribed to them.

4. Collaborating with the patient's family or relative to promote appropriate use of the antibiotic.

5. Continue reviewing the latest local and international clinical practice guidelines.

6. Practice good hand-washing techniques to reduce risk of getting or spreading an infection-hands should be washed with warm water and soap for at least 20 seconds, and ensuring hands, instruments, and environment are clean.

7. Antibiotics should be prescribed for bacterial infection, and do not prescribed for viral infection.

8. Minimize the use of broad spectrum antibiotic unless for no alternative antibiotics from narrow spectrum antibiotic such as Fluroquinolones.

9. In case of severe infection, it is recommended to start with a broad spectrum antibiotic and switch to a narrow spectrum drug as soon as the bacterial infection is identified.

10. Starting with a broad spectrum antibiotic and switch to a narrow spectrum drug as soon as the bacterial infection is identified and according to local guidelines. 
Citation: Alanazi MQ (2018) Strategies to Enhance Antimicrobial Resistance Stewardship. J Antimicrob Agents 4: 156. doi:

11. Report cases of antibiotic-resistant to surveillance teams.

\section{Recommendation for community pharmacists}

a. Guidance patients on appropriate use of antibiotic, when prescribed.

b. Warning patients on antibiotic resistance.

c. Inform patients on adverse effects.

d. Never dispensed antibiotic without official prescription.

\section{Recommendation for institution}

Institutions may avoid the spread of antibiotic resistance by the following:

1. Influence healthcare professionals to prescribe appropriate antibiotics

2. Educate and inform patients and healthcare professionals about the necessity of preventing resistance to antibiotics

3. Cultivate the skill of assessing medical condition, type of infection, and type treatment.

4. Creation of Committees to formulate policies and procedures in handling antibiotic resistant patients.

5. Restrict prescribing antibiotic

6. Local guidelines for the utilization antibiotic in hospital based on sensitivity of antibiotic

7. Update guidelines annually

8. Update the sensitivity and resistance of microbiology.

9. Awareness antibiotic day for healthcare providers and patients.

10. Development the surveillance of antibiotic-resistant infections.

11. Support policies, programs, and implementation of infection prevention and control measures.

\section{Conclusion}

Educating patients is the best way to minimize treatment of illness with the intake of antibiotic wherein there is a greater chance to develop antibiotic resistance. Other additional safety procedures to support better management against antibiotic resistance are appropriateness in prescribing antibiotic, obtaining an accurate diagnosis of the infection, understanding the difference between empiric and definitive therapy, detecting circumstances to switch to narrow-spectrum, cost-effective oral agents for the shortest duration necessary and understanding drug features that are uncommon to antibiotic agents.

\section{References}

1. Lacy C, Lance LL, Armstrong LL (2017) Drug Information Handbook. APhA (26th Edn).

2. Centres for disease control and prevention (2017) Emerging infect dis 24: 1-196.

3. Leekha S, Terrell CL, Edson RS (2011) General principles of antimicrobial therapy. Mayo Clin Proc 86: 156-167.

4. Adzitey F (2015) Antibiotic classes and antibiotic susceptibility of bacterial isolates from selected poultry; A mini review. World Vet J 5: $36-41$.

5. Srivastava A, Talaue M, Liu S, Degen D, Ebright RY, et al. (2011) New target for inhibition of bacterial RNA polymerase: 'Switch region'. Curr Opin Microbiol 14: 532-543

6. Schwalbe R, Moore LS, Goodwin AC (2007) Antimicrobial susceptibility testing protocols. Pp. 1-432.

7. WHO (2001) Global strategy for containment of antimicrobial resistance.

8. Uchil RR, Kohli GS, Katekhaye VM, Swami OC (2014) Strategies to combat antimicrobial resistance J Clin Diagn Res 8: ME01-ME04.

9. Alanazi MQ, Al-Jeraisy MI, Salam M (2015) Prevalence and predictors of antibiotic prescription errors in an emergency department, Central Saudi Arabia. Drug Healthcare Patient Saf 7: 103-111.

10. Alanazi MQ (2017) Impact of computerized physician order entry on medication errors and cost. Research and Reports in Medical Science 1:1

11. Wise R, Hart T, Cars O, Streulens M, Helmouth R, et al. (1998) Antimicrobial resistance is a major threat to public health. BMJ 317 : 609-610.

12. Levy SB (1994) Balancing the drug-resistance equation. Trends Microbiol 2: 341-342.

13. World Health Organization (2012) The evolving threat of antimicrobial resistance. Options for action. GPS PUBLISHING, France.

14. Centre for Disease Control and prevention (2013) Antibiotic resistance threats in the United States, US Department of Human and Health Services 1-114.

15. ECDC Joint Technical Report (2009) The bacterial challenge: Time to react. Europ Centre Diseas Prevent Cont.

16. Broom B, Broom J, Kirby E, Plage S, Adams J (2014) What role do pharmacists play in mediating antibiotic use in hospitals? A qualitative study. BMJ Open 5: e008326.

17. Fleming N, Barber S, Oredope DA (2011) Pharmacists have a critical role in the conservation of effective antibiotics. Pharma J 2011: 2-10. 